\title{
On the qualitative properties in system dynamics models*
}

\author{
JAVIER ARACIL ${ }^{1}$
}

\begin{abstract}
This paper proposes that the concept of 'qualitative' developed around the qualitative theory of nonlinear dynamical systems be applied to the field of system dynamics in order to provide a more solid foundation for the use of the qualitative in this field. The advantages of the use of qualitative concepts are analyzed both from a conceptual and an operational point of view. A preliminary stage in the world modeling process is used to illustrate this proposal.
\end{abstract}

\section{Introduction}

This paper is intended to address the need for an improved understanding of the qualitative properties of system dynamics models. To that end, the concept of qualitative in the domain of system dynamics will be stated on a rigorous base. The concept of qualitative has had different connotations in that field. For instance, it has been alleged that the information contained in influence (or causal) diagrams has this nature (Wolstenholme, 1990). This is not the meaning of qualitative to be discussed here. The meaning to be discussed here is concerned mainly with dynamic tendencies: whether the system is growing, declining, oscillating, or in equilibrium. Qualitative, in that sense, has an fuzzy meaning that covers those aspects that are less well defined than the strictly quantitative aspects, but which characterize the system's behavior patterns.

System dynamics is both a mathematical modeling and a computer simulation methodology. As a modeling method, it focuses on the complex structure of the aspect of reality being modeled. This complexity is due to multiloop feedback substructures, nonlinearities, and delays. The consideration of these characteristics leads to mathematical models that are quite difficult to deal with with analytical tools. Therefore, a conflict is produced between mathematical analysis and computer modeling. This conflict has been solved in system dynamics with the use of computers. The computer is invoked as the solution to work with the models. One of the aims of this paper is to argue against the

\footnotetext{
* This paper is based on the material presented by the author at the "Journées Dynamique des Systèmes" held in Marseille on November 16-17, 1995.

${ }^{1}$ Escuela Superior de Ingenieros, Universidad de Sevilla, Camino de los Descubrimientos s/n, Sevilla41092 Spain. E-mail: aracil@cartuja.us.es.
} 
sole use of computers, showing that mathematical analysis holds benefits for system dynamics which have been neglected. If a mathematical language is used, the consequences of that usage ought to be fully accepted. A system dynamics model is a mathematical object and as such it deserves mathematical analysis.

The main source of information to build a system dynamics model is the mental model of the experts who are familiar with the system modeled. These mental models have a qualitative nature and are expressed in ordinary language to begin with. One of the most interesting contributions of system dynamics is a methodology to translate models expressed in the experts' common language into the mathematical object known by mathematicians as a dynamical system. Due to the complexity of dynamical systems, their behavior patterns are obtained by computer simulation, which is a hard quantitative technique. Then, in the first step of the methodology, the qualitative contents of the mental models are converted into quantitative form. Later, the dynamical system is computer processed to obtain the behavior patterns, again in quantitative form. And finally, the quantitative trajectories of the system are qualitatively interpreted retaining mainly the qualitative patterns: growth, decline, oscillation, etc. These two reversal steps are not fully satisfactory from a methodological point of view (see the argument between Forrester, Howard, and Holt partially reproduced in (Meadows, 1980, p. 53-54)). In any case, the relevance of the qualitative in the system dynamics context is clear. However, a rigorous treatment of this aspect of the qualitative is still lacking.

One way to deal accurately with the qualitative in the domain of dynamical systems is to use the results of the qualitative theory of nonlinear dynamical systems (Hale and Koçak, 1991; Kuznetsov, 1995). These results give rise to the qualitative analysis of dynamical systems. That theory supplies some concepts and tools to deal with the kind of problems just stated. Concepts such as structural stability, and tools such as state portrait or bifurcation analysis allow us to deal accurately with some of the qualitative problems raised in the last paragraph. All the problems cannot be solved, but the use of qualitative theory of dynamical systems is one of the approaches available to the system dynamicist to overcome the problems raised by the representation of qualitative properties. Some attempts to apply these results to system dynamics have been described (Aracil, 1979; 1981; 1984; Mosekilde et al., 1988). In addition to these references, the system dynamics analysis carried out by (Rasmussen et al., 1985; Brons and Sturis, 1991) on the economic long wave has also this nature. A thorough qualitative analysis of the "limits to growth" system dynamics archetype was published recently (Aracil et al., 1997), in which the behavior known as delayed catastrophe (Karsky, 1992) is thoroughly analyzed, providing insight into an apparently counterintuitive behavior. The present paper complements that one.

The contributions developed around the branch of artificial intelligence known as qualitative reasoning (Fishwick and Luker, 1991), and particularly those by Simon (Kalagnanam and Simon, 1991) may also be mentioned. However, that line of research focuses more on computer science methods than on mathematical analysis; here, the latter will be emphasized.

All the above references are addressed mainly to what could be called the operational side of qualitative analysis; that is, state portraits, bifurcations, etc. There is also a conceptual side which is inspired in the work of the french mathematician René Thom 
(Thom, 1972), and developed by E.C. Zeeman (Zeeman, 1977). Issues such as qualitative equivalence and systems morphology are developed around these contributions. The concept of morphology plays a central role in Thom's modeling philosophy, and is closely related to the approach presented in this paper. The goal of these concepts is to give some insight into the forms of system behavior, and to classify archetypical forms on the basis of qualitative properties. The morphology of a system is correlated to what is known in system dynamics as reference modes. A specific example of this will be given later. The Thom approach to system modeling can be considered an attempt to give an analogy theory. An essay to incorporate these ideas into system dynamics modeling can be found in Aracil (Aracil, 1986).

In this paper, the thesis will be proposed through the analysis of a concrete system dynamics model. To that end, a preliminary version of the world model was chosen. This model is reported in Randers (Randers, 1974; 1980). It is a two-level model (excluding a delay) and is proposed as a simplified version of the modeling work described in the renowned limits to growth report (Meadows et al., 1974). The model is not fully documented in these references, but in Randers (Randers, 1974) there is enough information to reproduce it. As will be seen, this simple model is able to produce the main behavior modes the modelers wanted to include in their model. In this way, it supplies a very elementary example of a system representation capable of generating the qualitative behavior the modelers aimed to reproduce. However, the model was considered only an initial step in the modeling process, not studied in depth, and to a certain extent forgotten (it was not even named, so here it will be called World0). It seems that it was considered as a preliminary sketch of the true painting, which is the World3 model (Meadows, 1974). However, modern art has taught us that sketches, even if apparently unfinished, cannot be rejected, and they can be even more expressive and valuable than fully completed paintings.

The World0 model can be easily analyzed mathematically and simulated by computer due to its simplicity. Therefore, it supplies a specific example of this paper's main point: the need for complementarity between computer simulation and mathematical analysis with the tools supplied by the modern theory of nonlinear dynamical systems. This last theory is particularly concerned with the qualitative aspects of the system's behavior, and it will prove to be very well suited to the need that arises in system dynamics for a solid base for its qualitative claims. These should be distinguished from the strictly quantitative ones. According to the quantitative modeling process, the process itself should be reduced to the equation: data $\rightarrow$ model (Kalman, 1980). However, in system dynamics, the mental model is at the root of the modeling process, and that model is more qualitative than quantitative. This will be clarified below with the World0 model.

The paper is organized as follows. To begin with, in the next section, some problems raised by the representation of qualitative properties in system dynamics are discussed. In Section 3, the model World0 to be analyzed is described. Then the kind of analysis proposed is applied to this model, showing how a global perspective on the behavior modes of the system is reached. A section of reflections follows, where the general relevance of the analysis developed is pointed out. The paper ends with a section of conclusions. 


\section{The representation of the qualitative in system dynamics}

World modeling has been one of the most popular system dynamics endeavors. In recent years, it has led to a new report (Meadows et al., 1992) where the main recommendations of the earlier 1972 limits to growth study (Meadows et al., 1974) were updated. Both reports are based on the World3 system dynamics model, which is a medium-size model. Page 105 of Beyond the Limits states: "What we mean by 'real world' or 'reality' are the mental models of the authors of this book.' This is a very fair and honest statement of the point of departure of every modeling process. It clearly states the central role of the mental models, and at the same time shows the qualitative roots of the modeling framework. A few pages later (on p. 108), one can read: "The art of modeling... is to include just what is necessary to achieve the purpose, and no more.' The next page continues: "The purpose - the only purpose - of World3 is to understand the possible modes of approach of human economy to the carrying capacity of the planet. It is for that purpose only that we believe the model is "valid'." Then the main goal is also clearly stated: to reproduce the behavioral patterns by which human economy approaches the carrying capacity of the planet earth. These patterns are summarized in Figure 1, and are reproduced here for the sake of completeness. These figures show the behavior patterns that interested the modelers. It should be noted that in this case the morphology of the behavior patterns can be expressed through some simple geometrical objects. Significantly, this same figure appears in both reports (Meadows et al., 1974, p. 8) and (Meadows et al., 1992, p. 108).

As mentioned above, Randers has given a reduced (two level) version of the World3 model which, notwithstanding, generates the behavioral patterns of Figure 1. This is the model which will be analyzed in the sequel. This last model is based on the mental model described in a verbal form with four statements (Randers, 1974; 1980):

- "Human activity expands when there is unutilized capacity;

- a sufficiently high level of human activity erodes the carrying capacity;

- population does not respond by deliberately reducing an excessive load until a delay, during which time is spent in data gathering and institutional change;

- exceeding the carrying capacity forces an involuntary downward pressure on human activity - for example, through starvation.',

This set of qualitative statements is given as the basis of the modeling process. It should be realized that the modelers did not prove that world evolution must obey these statements. Rather, they constructed a model for such evolution, and argued that the model would best reproduce the behavior patterns they expected. The influence diagram in Figure 2 and the system dynamics diagram in Figure 3 were constructed based on these statements. They lead to a system dynamics model (see Appendix), which produces trajectories that fit in well with the main patterns of Figure 1 that is, which meet the leading qualitative goal of the modeling process.

A point that deserves some consideration is the qualitative nature of the concepts involved in the modeling process. This process begins by singling out some variables associated with the verbal description of the mental model. Following the above verbal 


\section{STABLE}

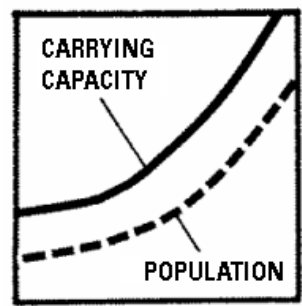

CONTINUOUS GROWTH

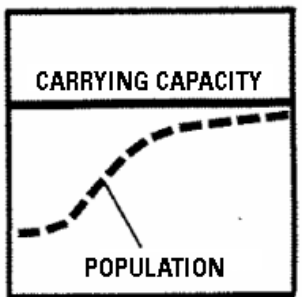

SIGMOID APPROACH TO EQUILIBRIUM

\section{UNSTABLE}

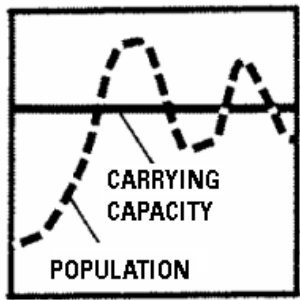

OVERSHOOT AND OSCILLATION

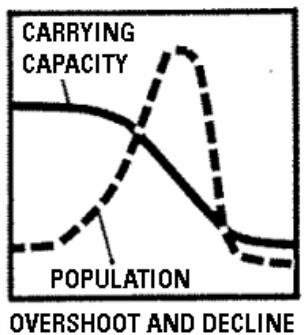

Fig. 1. Behavior patterns of world modeling; (Reproduced from Meadows et al., 1992, all rights reserved).

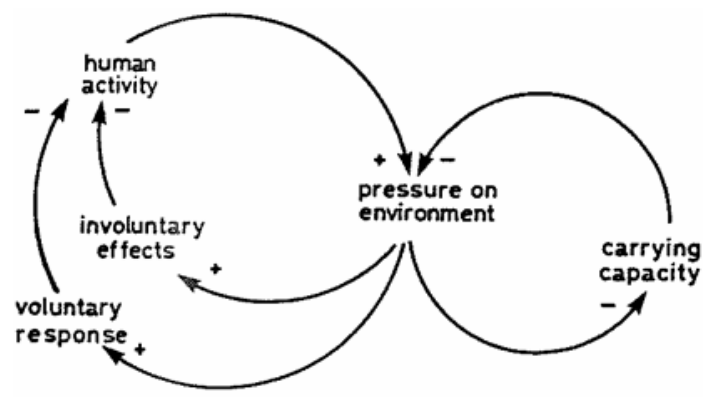

Fig. 2. Influence diagram of the reduced two-level world model (Reproduced from Randers 1980, all rights reserved).

description, two main system descriptors, 'human activity' and 'carrying capacity,' and one secondary descriptor associated with a delay, the 'voluntary response to pressure', were adopted in the World0 model (Fig. 3). The qualitative nature of these descriptors should be remarked. However, it is possible to state this meaning on a rigorously qualitative foundation. This is a very crucial methodological problem which at least in principle can be tackled with the concept of qualitative proposed by Zeeman (Zeeman, 1977, pp. 319 and ff.). 


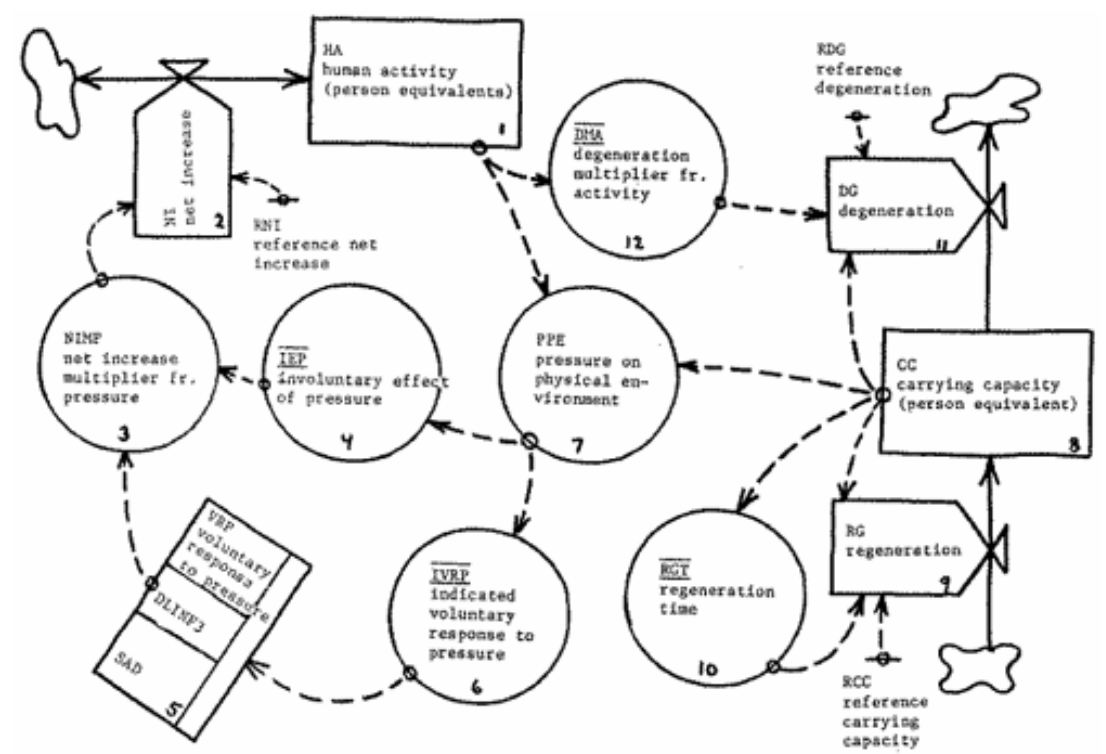

Fig. 3. System dynamics diagram of the reduced two-level world model (Reproduced from Randers 1980, all rights reserved).

There is no single way to measure 'human activity' (and some other of the concepts involved in the modeling process). It seems unreasonable to try to find a quantitative agreement on their meaning. Different modelers will measure 'human activity' according to their own diverse theories and methods, giving different quantitative measures of that system descriptor. This lack of uniqueness lends 'human activity' its qualitative nature. According to the Zeeman's definition of qualitative, all modelers must agree on a basic condition for their measurements: the change of scale for that quantity between different modelers has to be smooth and order-preserving. That means that any change of scale must be represented by a topological transformation which is non-linear, but monotone increasing. These transformations can be thought of as deforming the variables by stretching or squeezing them, but without folding or tearing. Consequently, the qualitative nature is associated with some "plasticity" of the concepts involved. The crucial point here is to realize that when dealing with concepts such as 'human activity', these transformations are associated with the qualitative nature of that concept. Under these transformations, order relations are preserved but not algebraic operations.

According to Zeeman's concept of qualitative, a conclusion that is valid in every measurement scale is said to be qualitatively invariant, or a qualitative conclusion (Zeeman, 1977, p. 321). That means that these properties are invariant under topological transformations of the system variables.

In the context of Zeeman's concept of qualitative, a distinction can be made between strong qualitative equivalence and weak qualitative equivalence. The former is attained when every monotone transformation compatible with the monotony assumption is allowed. This is a very strong assumption, as one clearly sees bearing in mind "all" the transformations that are monotone increasing. This is an extremal situation which is 
contemplated in methodologies such as the qualitative simulation introduced by Kuipers (Kuipers, 1994), where all the influences among variables have this nature. This is not reasonable for the use normally given to the qualitative in system dynamics. It is easy to see that this requirement is too restrictive in the last domain. A weaker assumption will be adopted here, where only small deformations will be allowed to cope with the imprecision of the concepts. The weak qualitative equivalence only considers small deformations around a nominal value. The range of scales for coping with the qualitative nature of a concept should be thought of as a fuzzy band around the identity map. In system dynamics, this kind of weak qualitative characterization is tacitly assumed.

The Zeeman's definition meets some general intuitive criteria. It is clear that if a trajectory is stretched or compressed as a result of a monotone transformation of the evolving variable, its shape remains unchanged (think of a graph trajectory drawn on a piece of rubber). Only the conclusions preserved after this type of deformations will be accepted. Thus, properties such as whether a given variable is growing or oscillating, or if a model has a certain kind of equilibrium, are legitimate conclusions which may be shared by different models. The same happens if the state portrait is subjected to this type of transformation on every axis. That means that two models are qualitatively similar if the state portrait of one of them can be obtained from the other by a continuous transformation.

In the context of the qualitative theory of nonlinear dynamical systems, qualitative properties are those properties of the state portrait which are topological invariants. A qualitative conclusion is shared by all the models belonging to the same class of qualitatively equivalent systems (Kuznetsov, 1995, p. 36 and ff.). Every model of a given equivalence class displays a behavior pattern that is representative of the whole class. One of the main qualitative properties of a dynamical system is that the existence and stability of an equilibrium point are invariant under monotonic transformations. Qualitatively equivalent models have the same number of equilibria and limit cycles of the same stability types; that is, the same long-term qualitative behavior.

The implication of this characterization of the qualitative is that, although qualitatively equivalent models may produce different quantitative trajectories, only the qualitative properties of these graphs can be considered admissible conclusions. It is interesting to realize that with this formalization of the qualitative, not only is the qualitative nature of the system descriptors stated on a rigorous foundation, but it is also incorporated into the dynamical systems that the models are.

\section{An example of qualitative analysis: world modeling}

Now let us come back to the World0 model. Making the 'human activity' $\mathrm{HA}=x_{1}$, the 'carrying capacity' $\mathrm{CC}=x_{2}$, and the 'voluntary response to pressure' $\mathrm{VRP}=z$, the model can be written by the dynamical system (see Appendix)

$$
\begin{aligned}
x_{1} & =k_{1} x_{1}\left[f_{1}\left(x_{1} / x_{2}\right)+z\right] \\
x_{2} & =\left(a-x_{2}\right) / f_{3}\left(x_{2} / a\right)-b x_{2} f_{4}\left(x_{1} / x_{10}\right) \\
z & =\operatorname{delay}_{\tau}\left(f_{2}\left(x_{1} / x_{2}\right)\right)
\end{aligned}
$$



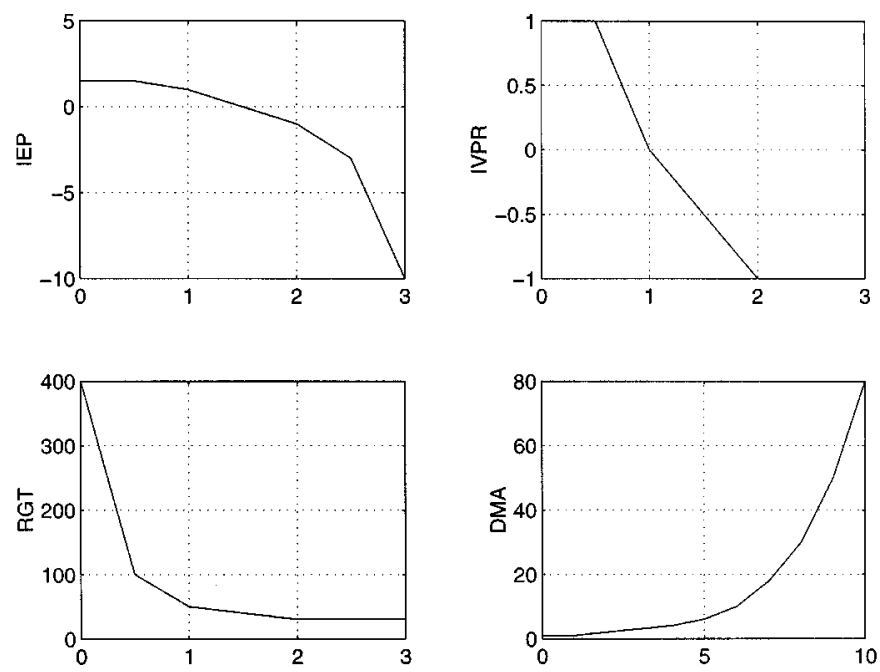

Fig. 4. Table functions in the World0 model: $I E P=f_{1} ; I V R P=f_{2} ; R G T=f_{3} ;$ and $D M A=f_{4}$.

where $x_{10}$ is the initial value of $x_{1}, k_{1}=\mathrm{RN}, a=\mathrm{RCC}, b=\mathrm{RDG}, f_{i}$ are the tables (nonlinearities) of the system and are shown in Figure 4, and the delay function returns $_{\tau}$ $\operatorname{delay}_{\tau}(y(t))=y(t-\tau)$. This last delay is approximated by a third-order delay. The system is defined only for $x_{1}, x_{2}>0$.

The first step in qualitative analysis is to find out the equilibria of the system. In the long range, for $t \rightarrow \infty$, the effect of the delays on stable equilibria disappears. This means that the equilibria of the above system are the same as of the system

$$
\begin{aligned}
& x_{1}=k_{1} x_{1}\left[f_{1}\left(x_{1} / x_{2}\right)+f_{2}\left(x_{1} / x_{2}\right)\right] \\
& x_{2}=g\left(x_{1}, x_{2}\right)
\end{aligned}
$$

where $g\left(x_{1}, x_{2}\right)=\left(a-x_{2}\right) / f_{3}\left(x_{2} / a\right)-b x_{2} f_{4}\left(x_{1} / x_{10}\right)$. The equilibria are defined by the condition $\dot{x}_{1}=\dot{x}_{2}=0$. Therefore the equilibria are the points defined either by $x_{1}=0$ and $g\left(x_{1}, x_{2}\right)=0$; or by $f_{1}=-f_{2}$ and $g\left(x_{1}, x_{2}\right)=0$.

To determine the equilibria, a graphical procedure will be used. Points $\left(x_{1}, x_{2}\right)$ satisfying condition $g\left(x_{1}, x_{2}\right)=0$, are points such that

$$
\frac{1-x_{2} / a}{f_{3}\left(x_{2} / a\right) x_{2} / a}=b f_{4}\left(x_{1} / x_{10}\right) \text {. }
$$

It is better to work on the normalized plane $\left(\bar{x}_{1}, \bar{x}_{2}\right)$ instead of $\left(x_{1}, x_{2}\right)$, where $\bar{x}_{1}=x_{1} / x_{10}$ and $\bar{x}_{2}=x_{2} / a$. Then the last equation is rewritten as

$$
\frac{1-\bar{x}_{2}}{f_{3}\left(\bar{x}_{2}\right) \bar{x}_{2}}=b f_{4}\left(\bar{x}_{1}\right) \text {. }
$$

This expresion leads to the graph shown in Figure 5. 


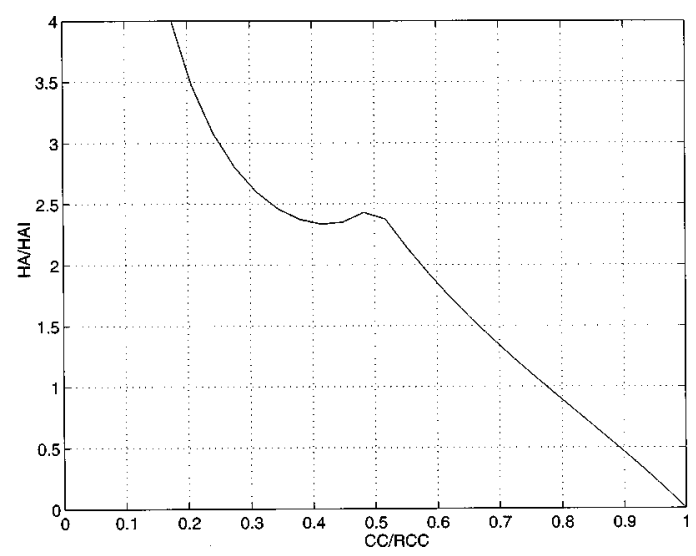

Fig. 5. Graphical representation of $g\left(\bar{x}_{1}, \bar{x}_{2}\right)=0$. The axes are scaled at the normalized variables $\bar{x}_{1}=H A / H A I$ (vertical axis) and $\bar{x}_{2}=C C / R C C$ (horizontal axis).

On the other hand, the condition $f_{1}=-f_{2}$ can be solved graphically as shown in Figure 6. According to this figure at the points satisfying $f_{1}=-f_{2}$ one has $x_{1} / x_{2}=1.3$ ( 3 stands for $333 \ldots$ ); that is, these points are on the straight line $x_{1}=1.3 x_{2}$. With normalized variables $\bar{x}_{1}$ and $\bar{x}_{2}$ this straight line is written

$$
\frac{\bar{x}_{1}}{\bar{x}_{2}}=\frac{x_{1} / x_{10}}{x_{2} / a}=\frac{a}{x_{10}} \frac{x_{1}}{x_{2}}=\frac{10}{1.6} 1.3=8.3
$$

As stated above, the equilibria are the points at the intersection of the graph of $g\left(\bar{x}_{1}, \bar{x}_{2}\right)=0$ either with the straight line $\bar{x}_{1} / \bar{x}_{2}=8.3$, or with $\bar{x}_{1}=0$. According to the shape of $g\left(\bar{x}_{1}, \bar{x}_{2}\right)=0$ in this case, there is one equilibrium of each type. However only the equilibrium where the the graph of $g\left(\bar{x}_{1}, \bar{x}_{2}\right)=0$ intersects with the straight line $\bar{x}_{1} / \bar{x}_{2}=8.3$ will be of interest here, because the other one $\left(\bar{x}_{1}=0, \bar{x}_{1}=1\right)$ is at the boundaries of the region where the model is defined, and furthermore it results to be unstable. Later the effects of this unstable equilibrium will be seen on the state portrait, showing that it is not relevant for the qualitative analysis of World0 model.

Figure 7 shows the equilibrium for the nominal case at the crossing point of straight line $\mathrm{N}$ (representing $\bar{x}_{1} / \bar{x}_{2}=8.3$ ) and curve $\mathrm{G}$ (representing $g\left(\bar{x}_{1}, \bar{x}_{2}\right)=0$ ). The values of $x_{1}$ and $\bar{x}_{2}$ at the equilibrium (resp. $x_{1}$ and $x_{2}$ ) obtained from Figure 7 can be checked by simulation of the model. In that way values $\mathrm{CC}=3.112 \mathrm{~B}$ and $\mathrm{HA}=4.150 \mathrm{~B}$ are obtained in agreement with the previous analyses.

It is clear that the system's equilibria depend on the way the graphs of Tables $f_{1}$ and $f_{2}$ intersect each other. Then, one way to change the equilibrium point is to change these tables. That is what was done in (Randers, 1974), where some changes in social policies were implemented to get more desirable results. These changes lead Randers to modify table $f_{2}$. However, how these changes would cause these improvements remains unexplained in that reference. 


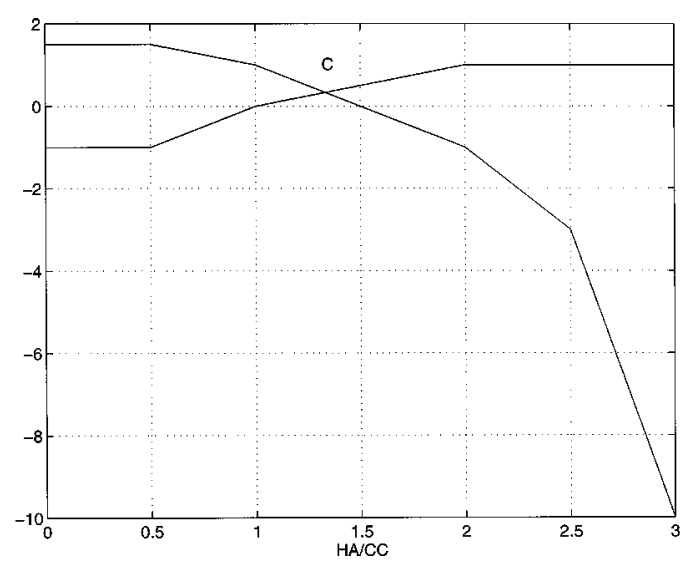

Fig. 6. Solving $f_{1}-f_{2}$. At the crossing point $C, P P E=H A / C C=x_{1} / x_{2}=1.3$, and then $\bar{x}_{1} / \bar{x}_{2}=8.3$.

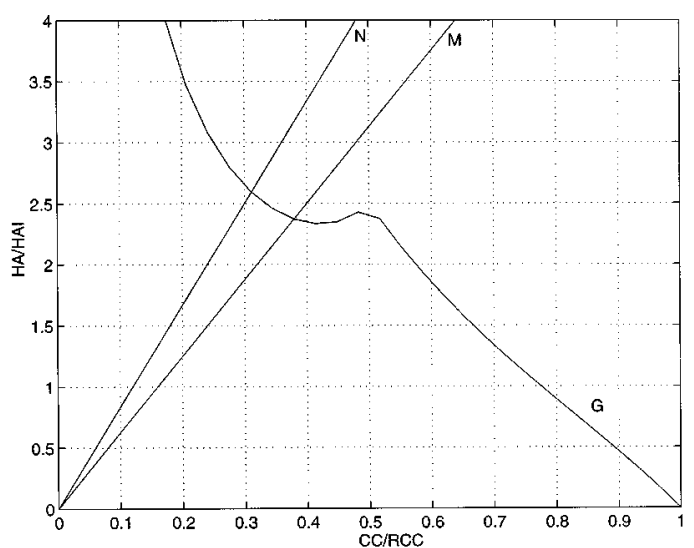

Fig. 7. Graphical determination of the equilibrium point: nominal case at the crossing point of straight line $N$ and curve $G$; and for the modified case at the crossing of $M$ and $G$.

Figure 8 shows the intersection of the $f_{1}$ with the new $f_{2}$ proposed in (Randers, 1974). It shows that the new value of $\mathrm{PPE}=\mathrm{HA} / \mathrm{CC}$ at the equilibrium is $\mathrm{PPE}=1$. That is, the value of PPE has diminished. That means, in Figure 7, that the slope of the modified straight line $\mathrm{M}$ is lesser than that of $\mathrm{N}$. Then, due to the shape of the graph of $g_{2}$, the value of $\mathrm{HA}$ at the equilibrium decreases, and at the same time the value of $\mathrm{CC}$ increases. If the model is run with the new $f_{2}$ table, the equilibrium is reached at $\mathrm{CC}=\mathrm{HA}=$ 3.795B. It should be realized that the above analysis shows that the best policy will be the one associated with the straight line that crosses the relative maximum of curve $g\left(\bar{x}_{1}, \bar{x}_{2}\right)$ in Figure 7. 


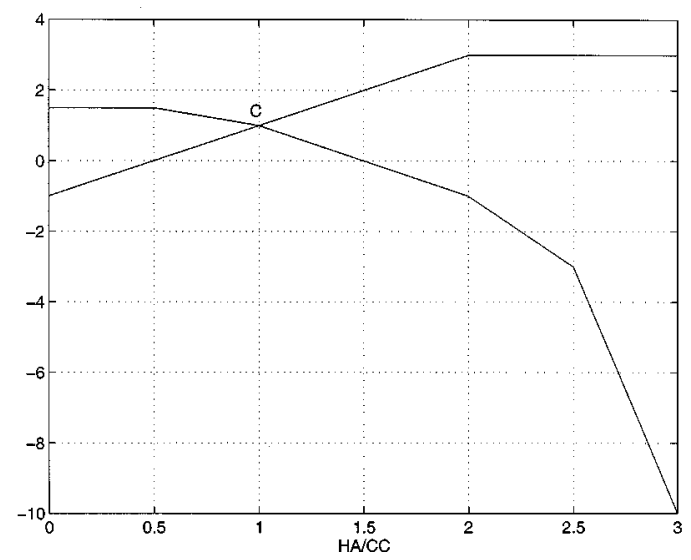

Fig. 8. Intersection of $f_{2}$ with $-f_{1}$ after changes in social policies.

Human activity is reduced with these new equilibrium values, but carrying capacity is less eroded. This was one of the explicit goals of the modelers. The method proposed here makes clear why this goal has been attained by changing the equilibrium point. Figures 6 and 7 show how to change $f_{2}$ (or $f_{1}$ ) to decrease the value of parameter PPE at the equilibrium, and then to reduce the slope of the straight line in Figure 7 to produce a change of the equilibrium point in the desired way. Now the mechanism to reach the goal of reducing the erosion of the carrying capacity has been made explicit. With this understanding, a huge number of changes in tables $f_{1}$ and $f_{2}$ can be conceived to produce similar effects. And in particular, the effects of the unexplained changes in $f_{2}$ proposed by Randers becomes clear. Even a better policy has been sugested.

In Figure 1, the behaviors were classified by the authors of the World3 as stable (the two at the top) and unstable (the two at the bottom). However, this classification does not fit the conventional uses in dynamical systems theory. As a matter of fact, all four behaviors are stable, as can be easily checked by running the model for a longer period (let's say 2000 years). Figure 1 only shows the transients but not the equilibrium points. As has been seen, in order to get better results in terms of less eroded carrying capacity (even if human activity is severely decreased) what ought to be done is to decrease the value of parameter PPE at the equilibrium (Fig. 7). Mathematically, this is due to the fact that the equilibrium is always stable for the non-delay case.

The other concern of the modelers was to manage the overshoot. The overshoot is related to the delay of the voluntary response to pressure. To study the effects of the delay, a qualitative method such as the one used in (Aracil et al., 1997) could be employed. The full development of that method for the current case does not fall within the scope of this paper. Only the main lines of analysis will be given. First, consider the system without delay given by equations (4) and (5). The equilibria of this system are the same as of the system with delay given by equations (1), (2) and (3). The way the equili- 
bria of the system given by equations (4) and (5) are reached is governed by the Jacobian matrix:

$$
J=\left[\begin{array}{cc}
k_{1} \frac{x_{1}}{x_{2}}\left[f_{1}^{\prime}+f_{2}^{\prime}\right] & -k_{1}\left(\frac{x_{1}}{x_{2}}\right)^{2}\left[f_{1}^{\prime}+f_{2}^{\prime}\right] \\
-\frac{b x_{2}}{x_{10}} & \frac{-a-b f_{4}\left(f_{3}^{\prime} x_{2}+a f_{3}\right)}{a f_{3}}
\end{array}\right]
$$

which for the concrete equilibrium point of Figure 7 , in the nominal case, leads to a matrix whose eigenvalues are $\lambda_{1,2}=-0.0139 \pm j 0.0127$. A complex value for $\lambda_{1,2}$ means that the equilibrium is reached with some oscillation, as is shown in the state space display of the trajectory $a$ in Figure 9.

Now consider the effects of the delay on the transient of this trajectory. The main problem in dealing mathematically with a delay is the infinite order of the dynamical system involved. In system dynamics, to overcome this difficulty, it is usual to approximate the delay with some first order systems in series. Then the equations of the model are written:

$$
\left\{\begin{aligned}
x_{1} & \left.=k_{1} x_{1}\left[f_{1}\left(x_{1} / x_{2}\right)+z\right)\right] \\
x_{2} & =\left(a-x_{2}\right) / f_{3}\left(x_{2} / a\right)-b x_{2} f_{1}\left(x_{1} / x_{10}\right) \\
z_{1} & =k a\left(f_{2}\left(x_{1} / x_{2}\right)-z_{1}\right) \\
z_{2} & =k a\left(z_{1}-z_{2}\right) \\
& \vdots \\
\dot{z_{k-1}} & =k a\left(z_{k-2}-z_{k-1}\right) \\
\dot{z} & =k a\left(z_{k-1}-z\right)
\end{aligned}\right.
$$

where the delay is $\tau=1 / a$. Usually $k=3$. Now there is a finite order system, which is easier to deal with with mathematical analysis tools. At the equilibrium $\dot{x}_{1}=\dot{x}_{2}=\dot{z}_{1}=\ldots=\dot{z}=0$, then $z=z_{1}=z_{2}=\ldots=f_{2}\left(x_{1} / x_{2}\right)$, and therefore the equilibrium is the same as without a delay. However, the eigenvalues, and eventually the stability, will change. For the case $k=1$, Figure 10 shows the root-locus of the system at the equilibrium as the time delay $\tau=1 / a$ varies. In this case, there are three loci because it is a third order system. Two of the branches start at the eigenvalues of the system without delay and the other at infinity. In the loci, it can be checked that for the current values of the delay the system tends to become more oscillatory as $\tau$ grows, since the complex roots tend to approach the imaginary axis. This is confirmed in the sequence of state portraits shown in Figure 9. The increase in oscillations is the cause of the corresponding growth of the overshoot, which is the second concern of the world system modelers. So to decrease the overshoot, the delay in the voluntary response has to be reduced as much as possible. The relationship between overshoot and delay has been made explicit. 


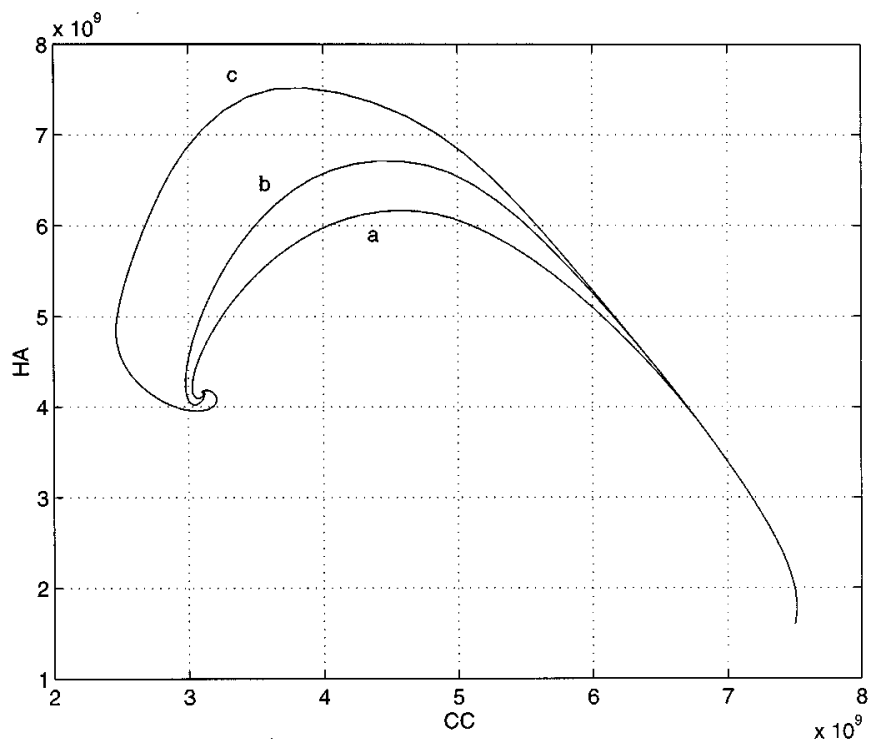

Fig. 9. Trajectories in the $\left(x_{1}, x_{2}\right)$ state space for different values of the delay: case a without delay; case $b$ with $\tau=30$; and case $c$ with $\tau=300$.

In this way, it becomes clear that a well-conceived policy applied with delay can undermine the success of the action. This raises a caveat: delays will deteriorate the crisis. An otherwise correct policy applied at a delayed time could result in catastrophe. In an accelerated world, this can lead to severe problems, particularly for large strategic issues. In any case, the previous analysis allows for a better understanding of the effects of response delay on behavior patterns. A more thorough examination of the underlying mathematical analysis can be seen in Aracil (Aracil et al., 1997).

It should also be pointed out that increasing the delay could make the equilibrium become unstable. However, for the normal values of the model, this will not happen, as shown in the root-locus of Figure 10. This can also be checked by running the model.

The state portrait of the World 0 model (in reality the projection of the state portrait onto the $\left(x_{1}, x_{2}\right)$ plane) is quite simple (Fig. 11). It consists of a single point attractor with the trajectories spiraling towards it. It should be remarked that the equilibrium $\left(\bar{x}_{1}=0\right.$, $\bar{x}_{2}=0$ or $x_{2}=10^{10}$ ) does not affect the main shape of the state portrait. As has been seen, the attractor point can be moved to a more desirable equilibrium by changing the tables and eventually the parameters. Furthermore, the spiraling can also be altered by changing the value of the delay, and then improving the overshoot (Fig. 9).

It is easy to realize that the full morphology in Figure 1 can be obtained by an even simpler model, where the nonlinearities are further simplified. This will lead to some changes in the World0 model as a result of the analysis developed. However, here the goal is only to analyze the World0 model as was built by the original modelers without modifying it. 


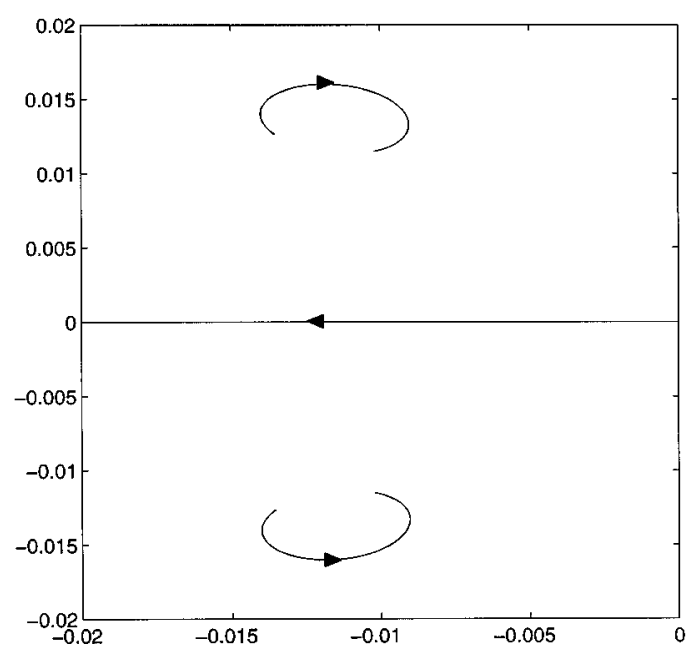

Fig. 10. Root-locus of the model World0 with a first order delay, as the delay $\tau$ varies. The arrows indicate $\tau$ increasing.

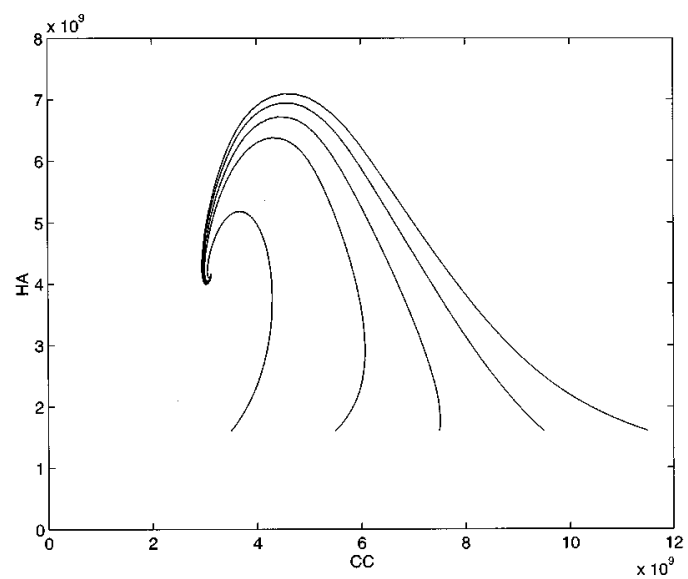

Fig. 11. Projection of the state portrait onto the $\left(x_{1}, x_{2}\right)$ plane.

\section{Some reflections on system dynamics modeling}

Model order is a very controversial point in world modeling. How many variables and parameters will be needed to fully characterize the world? Many sociologists and specialists in social sciences will argue that there is no finite number, and that if there were, it is beyond the powers of the human mind to tackle them. However, they usually make verbal statements about the world, trying to capture the essence of acquired knowledge about 
world evolution. The above four verbal statements of Randers mental model belong to this kind of knowledge and are organized in a mental model. The system dynamics model is just a translation of this type of mental model into the language of dynamical systems. Since the mental model only considers a limited number of statements, and therefore a limited number of elements of the aspect of reality considered, the system dynamics model based on it will also be of a limited order.

Taking into account the manifest goals of the modelers, one can argue whether there is no substantial difference between the model analyzed above (with two levels, excluding delays), or the World3 model (twelve levels) although the World0 model has not been refined enough. All of them share the same morphology summarized in Figure 1. Supporters of high-order models will assert that higher order models give more detailed information. This raises a traditional debate between the proponents of low-order models, and their detractors. The latter will also contend that customers ask for highorder models, which is true, as everyone engaged in system dynamics consulting knows. Nevertheless, it seems that strategic issues can be more soundly grounded with low-order models, and world modeling belongs to this class of models.

The confidence of a model user is reinforced when he becomes well-acquainted with the model contents and understands the behavioral implications of the mechanism that generates this behavior. The complexity of the higher order models may hide the behavior generation mechanism. Transparency has been one of the claims of system dynamics. However, the model should be transparent not only in its contents and documentation, but also in the way the mathematical mechanism generates the behavior patterns. This kind of transparency is better obtained with the simplest model that generates the pattern of behavior modes under study. One of the ground rules of scientific practice is Ockham's Razor, whereby hypotheses should not be introduced beyond the minimum number needed to explain the phenomenon to be modeled. For the modeling process, this means that the simplest model that accounts for the full behavior morphology under study should be adopted.

With the current qualitative analysis methods, the scope of applicability of qualitative analysis is restricted to low-order models. This can be thought of as an invitation to restrict oneself to such low-order models. Nevertheless, this can be unrealistic, at least outside of the academic world. But, in any case, qualitative analysis is also a valuable tool for large-scale models, because every system dynamics modeling process starts always with a low-order model. Then, understanding all the behavior patterns that elementary structure can generate should be very helpful for the peliminary steps of the modeling process.

The above comments underscore the relevance of some recent philosophical discusions about the problem of realism in natural and human sciences. More specificaly, Hilary Putnam's internal realism could offer a very interesting philosophical background for system dynamics, and in general for the modeling process (Vázquez et al., 1996a and 1996b). The role of the mental models and the variety of world modeling (not only the system dynamics ones) would put into question a number of very often assumed dichotomies such as the objective-subjective and the qualitative-quatitative ones. The point of view associated with Zeeman's qualitative scales, for instance, makes clear how the measurement belongs to the domain of the observer, or at least to the domain of the 
observer-observed interaction. Putnam's arguments against these dichotomies would fit very well with that sort of cases which show the many faces of realism (Putnam, 1987).

The last remark is about why the analysis techniques based on the qualitative analysis of nonlinear systems have not been more widely accepted in the system dynamics community. This is not an easy question to be answered. But in any case, it could be because of a "vicious circle": people do not use these analytical methods because they are not the appropriate tools in conventional system dynamics computer environments, and these environments do not include such tools because the common users do not ask for them. This cycle should be broken by software producers. With the current tools in system dynamics environments, qualitative analysis results are cumbersome. To obtain some of the results of the previous section, a general computer environment was used. For that, the model had to be translated from a system dynamics environment to that general one, which is not a very acceptable process.

\section{Conclusions}

The previous qualitative analysis of the World0 model leads to some reflections about system dynamics. First of all, system dynamics models are based on mental models, expressed in a verbal form, and the aims of the modeling process are also stated in a qualitative way. However, the computer model is a dynamical system that is processed as a quantitative object. The quantitative trajectories of the computer model are "read" qualitatively. It seems quite natural to use the tools of the qualitative theory of nonlinear dynamical systems throughout this process. This proposal should be understood as being complemented with computer simulation.

The benefits for system dynamics of the qualitative approach discussed in this paper are twofold: conceptual and operational. First, the use of qualitative concepts can be more soundly grounded using the Thom-Zeeman proposal. And even if this proposal is not fully accepted, the advantages for system dynamics of the qualitative analysis have a second class of benefits. These benefits include bringing an understanding of the mathematical mechanism which generates the system behavior, and helping to reach a global perspective on the behavior modes of the model, that can then be used as a guide for simulation. Understanding mathematical mechanism helps define policies to reach explicit goals. A global perspective on behavior modes allows us to look for appropriate leverage points.

Computer simulations alone do not provide a means for describing all the behavior modes of a dynamical system in a systematic way. For this global view, the complementary use of the results of the qualitative theory of nonlinear systems is proposed. Computer simulation and mathematical analysis are approaches that should be used cooperatively. Qualitative analysis must not be viewed as a goal in itself but as a basic step in system dynamics methodology.

To depend only on computer simulation, excluding the results of the mathematical theory of dynamical systems, compromises the acceptance of system dynamics as a wellgrounded discipline outside the established system dynamics community. The author of 
this paper maintains that this acceptance is deeply related to the reconciliation of mathematical analysis and computer simulation.

\section{Appendix}

In this appendix, the equations of the model are recalled. A more complete description of the model will be found in Randers (Randers, 1974). These equations, written in a conventional form, read:

$$
\begin{aligned}
\frac{\mathrm{d}(\mathrm{HA})}{\mathrm{d} t} & =\mathrm{NI} \\
& =\mathrm{HA} \times \mathrm{RN} \times \mathrm{NIMP} \\
& =\mathrm{HA} \times \mathrm{RN} \times(\mathrm{VRP}+\mathrm{IEP}) \\
& =\mathrm{HA} \times \mathrm{RN} \times\left(\mathrm{IVRP}_{\tau}+f_{1}(\mathrm{PPE})\right) \\
& =\mathrm{HA} \times \mathrm{RN} \times\left(f_{2}(\mathrm{PPE})+f_{1}(\mathrm{PPE})\right) \\
& =\mathrm{HA} \times \mathrm{RN} \times\left(f_{2}\left(\mathrm{HA}_{\tau} / \mathrm{CC}_{\tau}\right)+f_{1}(\mathrm{HA} / \mathrm{CC})\right)
\end{aligned}
$$

where $x_{\tau}$ stands for $x$ delayed $\tau$ times, that is $x_{\tau}(t)=x(t-\tau)$.

$$
\begin{aligned}
\frac{\mathrm{d}(\mathrm{CC})}{\mathrm{d} t} & =\mathrm{RC}-\mathrm{DG} \\
& =\frac{\mathrm{RCC}-\mathrm{CC}}{\mathrm{RGT}}-\mathrm{CC} \times \mathrm{RDG} \times \mathrm{DMA} \\
& =\frac{\mathrm{RCC}-\mathrm{CC}}{f_{3}(\mathrm{CC} / \mathrm{RCC})}-\mathrm{CC} \times \mathrm{RDG} \times f_{1}(\mathrm{HA} / \mathrm{HAI})
\end{aligned}
$$

The main variables are 'human activity' HA, 'carrying capacity' CC and 'voluntary response to pressure' VRP. The parameter values and the initial conditions are: $\mathrm{RNI}=1.6 \times 10^{9}$, SAD (the time delay $\left.\tau\right)=30, \mathrm{RCC}=10^{10}, \mathrm{RDG}=.004, \mathrm{HA}\left(t_{0}\right)=$ $1.6 \times 10^{9}$ and $\mathrm{CC}\left(t_{0}\right)=7.5 \times 10^{9}$. The tables are given in Figure 4 .

Acknowledgments. The author appreciates the comments of Margarita Vázquez, Francisco Gordillo, Manuel Liz and Enrique Ponce on a draft of this paper.

This work has been supported by the Spanish Ministry of Education and Culture under grant CICYT TAP97-0553.

\section{References}

Aracil J. (1979) Stabilité Structurelle et Dynamique des Systèmes, Journées Dynamique des Systèmes, Mons, Juin 14-16.

Aracil J. (1981) Structural Stability of low-order System Dynamics models, Int. J. System Science 12, pp. 423-441. 
Aracil J. (1984) Qualitative analysis and Bifurcations in System Dynamics Models, IEEESMC-14 (4), pp. 688-696.

Aracil J. (1986) Bifurcations and structural stability in the dynamical systems modelling process, Systems Research 3 (4), pp. 243-252.

Aracil J., Ponce E., Pizarro L. (1997) Behavior patterns of logistic models with delay, Mathematics and Computers in Simulation 44, pp. 123-141.

Brons M., Sturis J. (1991) Local and global bifurcations in a model of the economic long wave, System Dynamics Review 7(1), pp. 41-60.

Fishwick P.A., Luker P.A. (Eds.) (1991) Qualitative Simulation Modeling and Analysis, Springer-Verlag.

Hale J.K., Koçak H. (1991) Dynamics and Bifurcations, Springer-Verlag.

Kalagnanam J., Simon H., Iwasaki Y. (1991) The Mathematical Bases for Qualitative Reasoning, IEEE Expert, April, 11-19.

Kalman R. (1980) A System-Theoretic Critique of Dynamic Economic Models, International Journal of Policy Analysis and Information Sciences 4(1), pp. 3-22.

Karsky M., Dore J-Ch., Gueneau P (1992) De la possibilité d'apparition de catastrophes différés, Ecodecision, No. 6, Septembre, Montreal.

Kuipers B. (1994) Qualitative Reasoning: Modeling and Simulation with Incomplete Knowledge, MIT Press.

Kuznetsov Y.A. (1995) Elements of Applied Bifurcation Theoy, Springer-Verlag.

Meadows D.H. (1980) The Unavoidable A Priori, in J. Randers (Ed.), The Elements of the System Dynamics Method, The MIT Press, 23-57.

Meadows D.H. et al. (1974) Dynamics of Growth in a Finite World, Wright-Allen Press.

Meadows D.H., Meadows D.L., Randers J. (1992) Beyond the Limits, Earthscan, London.

Mosekilde E., Aracil J., Allen P. (1988) Instabilities and chaos in non-linear dynamic systems, System Dynamics Review 4(1), pp. 14-55.

Putnam H. (1987) The Many Faces of Realism, Open Court.

Randers J. (1974) Conceptualizing Models of Social Systems, D. Meadows (Ed.), Proc. Conf. on Methodological Aspects of Social Systems Simulation, Sukhumi, USSR, pp. 12-49.

Randers J. (1980) Guidelines for Model Conceptualization, in Randers (Ed.), The Elements of the System Dynamics Method, The MIT Press, pp. 117-139.

Rasmussen S., Mosekilde E., Sterman J.D. (1985) Bifurcations and chaotic behavior in a simple model of the economic long wave, System Dynamics Review 1(1), pp. 92-110.

Thom R. (1972) Stabilité structurelle et morphogénese, InterEditions, Paris.

Vázquez M., Liz M., Aracil J. (1996a) Knowledge and reality: some conceptual issues in the system dynamics modelling, System Dynamics Review 12(1), pp. 21-37.

Vázquez M., Liz M., Aracil J. (1996b) An Epistemological Framework for System Dynamics Modelling, Revue Internationale de Systémique 9(5), pp. 461-489.

Wolstenholme E.F. (1990) System Enquiry, Wiley.

Zeeman E.C. (1977) Catastrophe Theory, Addison-Wesley. 